\title{
Abordaje fisioterápico en la cirugía por cáncer de pulmón
}

\author{
Physical therapy approach in lung cancer surgery
}

\author{
L. González Doniz, R. Fernández Cervantes, S. Souto Camba, A. López García
}

\begin{abstract}
Resumen
El presente artículo es una revisión sobre la fisioterapia respiratoria y la rehabilitación pulmonar en el cáncer de pulmón. Para ello se han consultado diferentes fuentes bibliográficas encontrando que, así como su rol dentro de otros tipos de cirugía a nivel abdominal alto ya nivel torácico (p. ej., las cirugías de reducción de volumen) está plenamente definido y argumentado, en el caso de las resecciones quirúrgicas por cáncer de pulmón con frecuencia se realizan extrapolaciones de lo que ha demostrado ser beneficioso en otros procedimientos quirúrgicos. En el artículo se abordan aspectos epidemiológicos relativos al cáncer de pulmón en España, los tipos de cirugía más empleados para su tratamiento y la intervención de fisioterapia en los momentos prequirúrgico y posquirúrgico, en relación con los diferentes objetivos terapéuticos y alteraciones funcionales encontradas. Asimismo, para su elaboración, se ha contado con la experiencia clínica de los autores en este ámbito.
\end{abstract}

\begin{abstract}
This article is a review about Respiratory Physical Therapy and Pulmonary Rehabilitation in the treatment of lung carcinoma. For that, different resources have been consulted The role of respiratory physical therapy and pulmonary rehabilitation is clearly defined in the upper abdominal surgery and in other thoracic surgeries, such as lung volume reduction, but in the case of lobectomies and pneumonectomies extrapolations are often made from what have been shown beneficial in other surgery processes. The article tackles lung carcinoma epidemiological issues, the main surgeries employed and the physical therapy treatment in the pre-operative and the post-operative period in relation to the functional findings and the therapeutic aims. Also, it was taken into account the clinical experience o/ the authors in this area.
\end{abstract}

Palabras clave

Fisioterapia respiratoria; Cirugía torácica; Cáncer de pulmón; Complicaciones posoperatorias; Período posoperatorio; Período preoperatorio.

\section{Keywords}

Respiratory therapy; Lung surgery; Lung carcinoma; Post-operative complications; Post-operative period; Preoperative period

\section{Introducción}

El cáncer de pulmón es un problema contemporáneo consecuencia directa principalmente del aumento sustancial del consumo de tabaco. En los países occidentales, la enfermedad cardiovascular y el cáncer siguen siendo la primera y segunda causa de muerte respectivamente. En la Unión Europea el cáncer pulmonar es el tipo de neoplasia más importante, con el índice de mortalidad más alto en el hombre y ocupando el tercer puesto en la mujer después de los cánceres de mama y colón. La relación de incidencia entre sexos puede tener un valor medio 6: 1 (10: 1 en España). Desde el punto de vista cuantitativo, se ha observado un incremento en la incidencia del cáncer. Se prevé que para la segunda década del nuevo milenio, en la Unión Europea el número de casos crezca en un 49\% en personas mayores de 65 años, respecto a $1990^{1}$.

El carcinoma de pulmón afecta con más frecuencia a los lóbulos superiores, seguidos de los lóbulos inferiores. El pulmón derecho suele afectarse más que el izquierdo. Desde el punto de vista de su localización, los tumores se clasifican en centrales, localizados en los bronquios principales, lobares y 
segmentarios hasta la quinta generación bronquial, y en periféricos, afectando a los bronquios de 5. generación en adelante, a los bronquiolos y a los alvéolos ${ }^{2}$.

Desde un punto de vista histológico, existen cuatro tipos fundamentales de tumor pulmonar invasivo: el carcinoma de células escamosas o carcinoma epidermoide; el adenocarcinoma, dentro del cual se incluye al carcinoma bronquioloalveolar; el carcinoma anaplásico de células grandes; y el carcinoma anaplásico de células pequeñas, que incluye el tipo de "células en grano de avena". Los más frecuentes son el adenocarcinoma y el carcinoma epidermoide. El adenocarcinoma suele estar localizado más periférico en el pulmón, y metastatiza pronto y de manera extensa al pulmón, hígado, huesos, riñones y Sistema Nervioso Central. El carcinoma epidermoide se origina en el epitelio bronquial metaplásico; el 50-60 \% se localizan proximalmente o afectan al hilio. Suele crecer dentro de la luz bronquial, causando obstrucción precoz con neumonitis secundaria. Su crecimiento es relativamente lento, por lo que no suele metastizar de forma precoz. ${ }^{3}$.

\section{Tratamiento quirúrgico del cáncer de pulmón}

La resección quirúrgica completa de cáncer de pulmón ofrece a los pacientes la oportunidad de curación y se considera como único tratamiento o como parte de tratamiento de algunos estadios. Las opciones quirúrgicas más frecuentes son la lobectomía, consistente en la resección quirúrgica de un lóbulo canceroso entero y neumonectomía, la resección completa de un pulmón. En ambos casos el acto quirúrgico puede presentar diferentes vías de abordaje.

La toracotomía posterolateral se reserva para casos difíciles en los que, por ejemplo, una extensa área de acceso es necesaria. Es la más dolorosa de todos los procedimientos quirúrgicos porque hay una gran afectación muscular, con resección del trapecio, dorsal ancho, la porción baja del romboide, el serrato anterior, los intercostales y los erectores vertebrales. Se emplea un retractor de costillas para aumentar el especio intercostal y, en determinadas situaciones, puede ser necesaria la re- sección total o parcial de las costillas para mejorar la exposición del pulmón.

La toracotomía axilar, a menudo referida como lateral, se emplea en cirugías poco complicadas. El único grupo muscular seccionado son los intercostales. El abordaje de las lesiones de lóbulo superior se realiza desde el cuarto espacio intercostal, mientras que el de las lesiones del lóbulo medio e inferior se realiza desde el quinto (fig. 1).

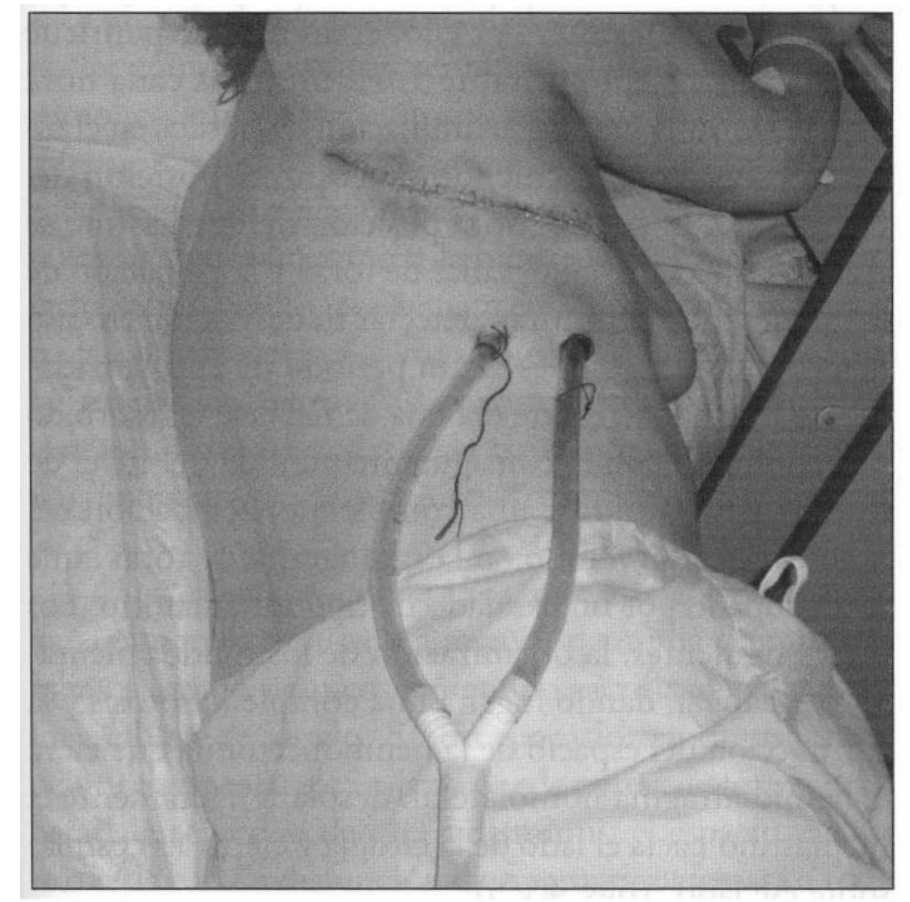

Fig. 1. Toracotomía lateral en $5 .^{\circ}$ espacio intercostal. 
La esternotornía media es poco usada, excepto en caso de resección de tumores bilaterales múltiples. Este tipo de incisión tiene su uso limitado por el difícil acceso a las estructuras hiliares posteriores del lóbulo inferior izquierdo.

Tras una lobectomía se suelen colocar dos drenajes intercostales en el espacio pleural, conectados a una succión continua, para evacuar el aire y el fluido/sangre del espacio vacante que deja el lóbulo reseccionado y favorecer la reexpansión del tejido pulmonar remanente que ocupará dicho espacio. Ocasionalmente se puede producir un ligero desplazamiento hacia arriba del hemidiafragma homolateral a la resección y del medias tino hacia el lado de la cirugía. Los drenajes se retiran cuando no hay escape de aire, visible por el cese de burbujeo en el depósito de agua de drenaje, o cuando se drenan menos de 50 ml. de fluido en 24 horas. El espacio pleural suele estar obliterado a la semana de la cirugía, si bien en algunos casos puede haber un escape persistente de aire que puede complicar el posoperatorio con un empiema o una fístula broncopleural (figs. 2 y 3 ).

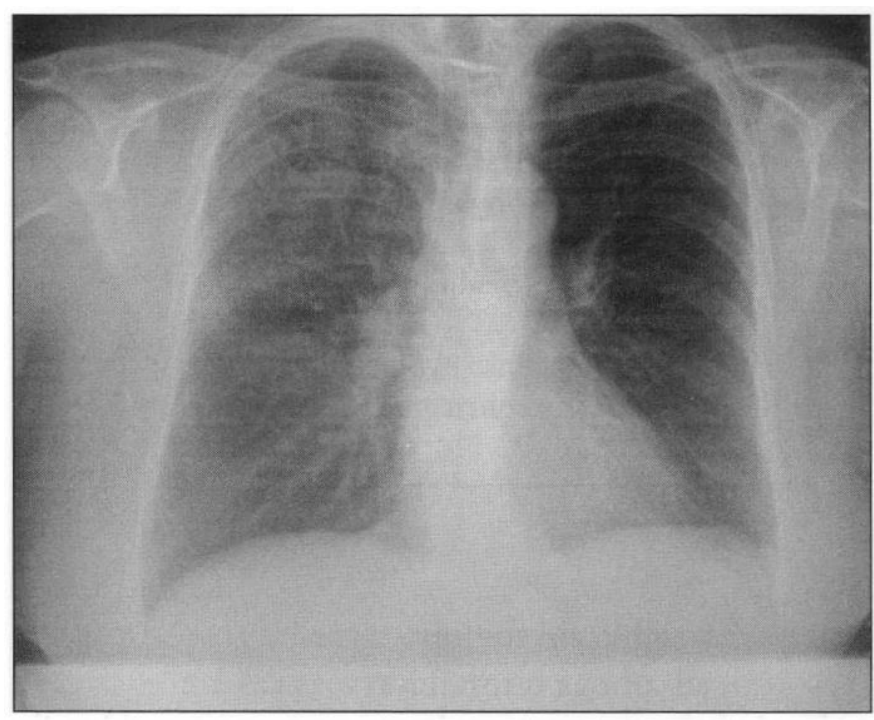

Fig. 2. Imagen prequirúrgica de un tórax con carcinoma epidermoide del lóbulo superior derecho.

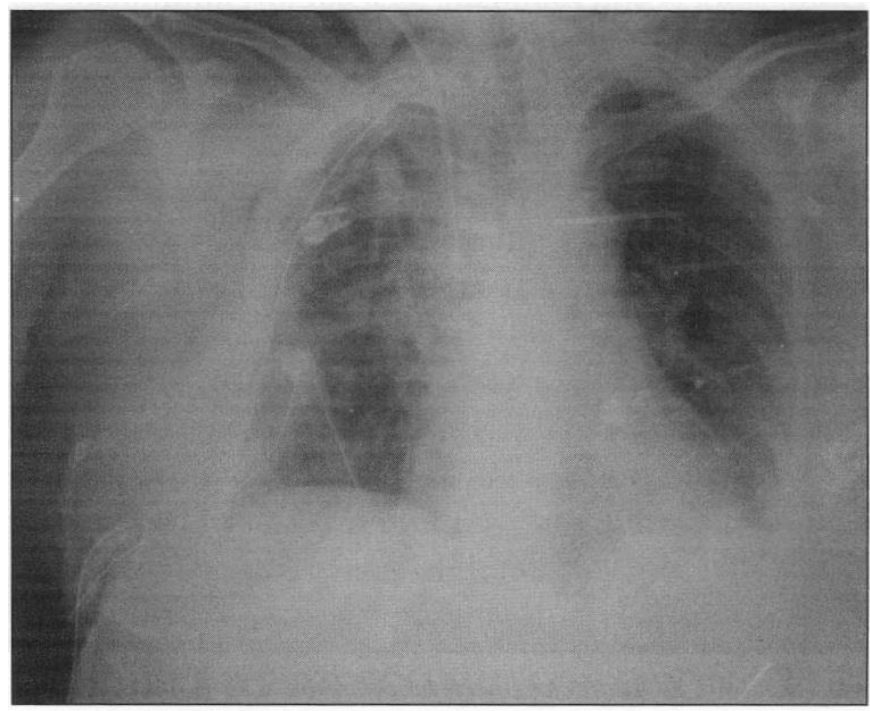

Fig. 3. Lobectomía del lóbulo superior derecho. Imagen en el posoperatorio inmediato, con observación de la pérdida de volumen pulmonar, de la elevación del hemidiafragma derecho y la presencia de los drenajes. 
En el caso de la neumonectomía, el espacio que deja el pulmón se ocupa con aire, sangre y fibrina, cuya cantidad es regulada a través de un único drenaje torácico. La relación que se establece entre el nivel de fluido y el aire que es absorbido (nivel hidroaéreo) determinará la posición del medias tino. Por ello, el drenaje se mantiene bloqueado excepto durante 1 o 2 minutos cada hora para permitir la gradual acumulación de fluidos en el espacio vacante, manteniendo una óptima posición del mediastino. El control de la posición del mediastino se realiza a través de radiografías de tórax y la palpación de la tráquea, que permitirán detectar su desviación en caso de producirse. Después de un período de aproximadamente 6 semanas, el aire debería de haberse reabsorbido por completo con la consiguiente pérdida del nivel de fluidos, observándose una completa opacificación del hemitórax intervenido en la radiografía de tórax, que está ocupado por un exudado de tipo inflamatorio. Los meses siguientes, la organización de los fluidos pleurales tiene lugar, dando lugar a una completa fibrosis y la contracción del espacio de la neumonectomía que eleva el hemidiafragma homolateral, desplaza ligeramente el mediastino hacia el lado de la cirugía y cierra las costillas sobre ese lado ${ }^{2}$ (figs. 4 y 5 ).

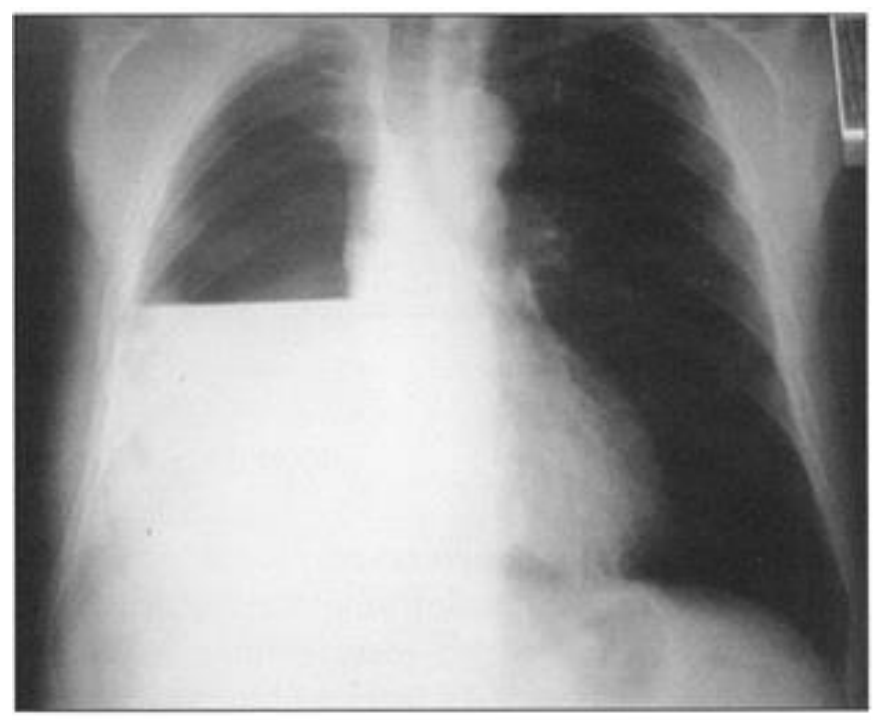

Fig. 4. Nivel hidroaéreo en una neumonectomía del pulmón derecho. Posoperatorio inmediato.

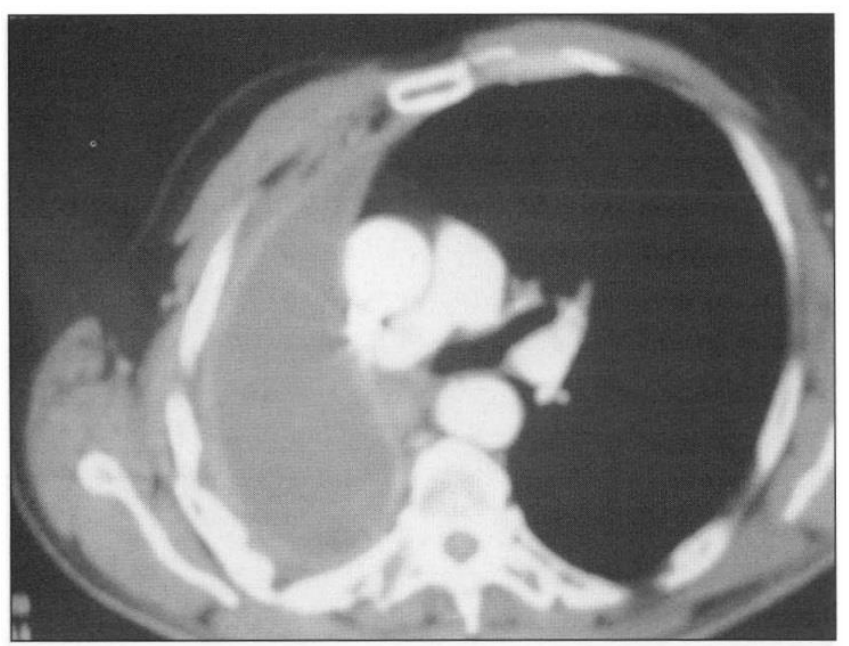

Fig. 5. TAC de una neumonectomía derecha a los 6 meses de la intervenci6n. Se observa el engrosamiento pleural con paquipleuritis derecha, la ocupaci6n del hemit6rax derecho por líquido fibrosado y el incremento del espacio aéreo izquierdo. 


\section{Complicaciones pulmonares de la cirugía de resección}

Se entiende por complicación pulmonar cualquier anormalidad pulmonar que ocurre durante el período posoperatorio y que produce una enfermedad o disfunción identificable y clínicamente significativa, que afecta de manera adversa al curso clínico de la misma ${ }^{4}$. Una verdadera complicación es considerada como un evento no esperado, que ocasiona síntomas y aumenta la morbimortalidad del paciente y por lo tanto prolonga el tiempo de estancia en la Unidad de Cuidados Intensivos (UCI), en el hospital e incrementa los costos de la atención del paciente. No deben considerarse complicaciones hallazgos posoperatorios esperados y por lo demás sin repercusión sobre los resultados fina- les, tales como la hipoxemia mínima, las caídas en los volúmenes pulmonares y la aparición de pequeñas atelectasias laminares. La tabla 1 presenta los criterios que se han de tener en cuenta en un posoperatorio, duran- te los primeros catorce días, para considerar la existencia de una complicación pulmonar clínicamente significativa. Se han de presentar 3 o más de los signos clínicos señalados 5

Tabla 1. Criterios complicación pulmonar

1. Cambios en la auscultación (disminución ruidos respiratorios, crujidos, sibilancias, respiración bronquial), a mayores de los presentes antes de la cirugía

2. Temperatura $>38^{\circ} \mathrm{C}$

3. Placa de tórax con cambios coherentes con colapso, consolidación o atelectasias

4. Incremento en la cantidad y/o cambio de coloración en la producción de esputo, comparado con la habitual en el enfermo

La cirugía de tórax es una de las que mayor incidencia de complicación presentan (10-40 \%). Verdaderas complicaciones posoperatorias serían las atelectasias y las infecciones respiratorias (bronquitis y neumonía), vinculadas a la disminución posoperatoria de los volúmenes pulmonares, las arritmias, la hipotensión y shock, el infarto de miocardio, el edema y embolismo pulmonar, el fracaso respiratorio, las fugas prolongadas de aire y dehiscencias, entre otras ${ }^{6}$, Las complicaciones pulmonares específicas derivadas de la cirugía de pulmón son las siguientes ${ }^{7}$ :

1. Disfunción diafragmática, que aparece cuando se daña el nervio frénico. La resección amplia de tumores hiliares o mediastínicos a menudo sacrifican de manera inevitable este nervio. En otras ocasiones, durante una neumonectomía, el cirujano puede cortar de forma deliberada este nervio de cara a disminuir el espacio vacan- te o residual. La disfunción diafragmática afectará a toda la mecánica ventilatoria y de la tos, favoreciendo las atelectasias posoperatorias.

2. La manipulación del pulmón para excluir la existencia de metástasis pulmonares puede conducir a la inflamación del tejido pulmonar ${ }^{2}$. Después de una lobectomía, la contusión en el pulmón adyacente puede causar alteraciones en la relación ventilación/perfusión (V/Q) e hipoxemia. Después de una neumonectomía, la hipoxemia aparece con frecuencia durante el ejercicio.

3. Las fugas de aire son una complicación previsible después de una lobectomía. El drenaje de tórax no se retira hasta que cese.

4. Enfisema subcutáneo. Sin repercusiones clínicas de relevancia, si bien es importante tranquilizar al paciente informándole sobre su carácter temporal.

5. Lesión del nervio laríngeo recurrente, especialmente presente después de neumonectomía izquierda o lobectomías de los lóbulos superiores. Su lesión puede afectar al habla y a la tos del paciente.

6. Fístula broncopleural, brecha entre el pulmón y la pleura debido a una rotura del extremo bronquial. Tiene el mismo efecto que un neumotórax. Su presencia contraindica las técnicas de fisioterapia con presión positiva.

7. El edema pulmonar post-neumonectomía puede aparecer en los tres primeros días del posoperatorio y se caracteriza por presentar cambios histológicos idénticos al síndrome de distrés respiratorio agudo. Es una complicación severa que normalmente precisa de la intubación del paciente ${ }^{2}$.

Las tasas medias de mortalidad de la resección de cáncer de pulmón son del $4 \%$, de modo que la lobectomía tiene una mortalidad de $3 \%$ y la neumonectomia del $7-9 \%$. Las causas más frecuentes 
incluyen la neumonía e insuficiencia respiratoria (41\%), infarto de miocardio(14\%), empiema y fístula broncopulmonar (11\%), hemorragias (7\%) y embolia pulmonar (6\%) 8 .

A la luz de estos datos, dos factores serán de gran relevancia para el control de las complicaciones posoperatorias y el éxito de las cirugías. Por un lado, la valoración del riesgo quirúrgico de manera previa a la cirugía, y por otro la intervención precoz de fisioterapia, como ya se ha demostrado con diferentes estudios realizados en pacientes quirúrgicos tóraco-abdorninales ${ }^{9,10}$.

\section{Factores de riesgo y valoración del riesgo quirúrgico}

Los factores de riesgo en relación con la aparición de complicaciones respiratorias son de dos tipos. Por un lado, factores de riesgo iniciales ligados al paciente, como son la malnutrición, las anomalías metabólicas, la obesidad, la edad, el tabaquismo y los antecedentes respiratorios. Por otro lado, están los factores de riesgo vinculados a la propia intervención, que supone la apertura de la pared torácica, la sección pleural, la amputación pulmonar, que conllevan a su vez una anestesia de tipo general, intubación endotraqueal y ventilación mecánica invasiva, junto con una posición determinada mantenida en la mesa de operaciones durante la cirugía.

a) Edad y obesidad: A pesar de que en la actualidad existen datos que no confirman que la obesidad y la edad tengan gran relevancia en el riesgo de complicación, se siguen teniendo en cuenta en la estratificación del riesgo ${ }^{6}$, ya que las condiciones de la función pulmonar de algún modo pueden estar alteradas desde el pun to de vista de la mecánica ventilatoria y por lo tanto influyen en la evolución posquirúrgica.

b) Tabaquismo y la Enfermedad Pulmonar Obstructiva Crónica (EPOC): El tabaco es un factor que aumenta claramente el riesgo de complicación posoperatoria, especialmente si la historia de tabaquismo excede los 20 paquetes-año o si el paciente ha fumado dentro de las 8 semanas previas a la cirugía ${ }^{11}$. Los fumadores suelen ser con gran frecuencia personas hipersecretantes y con un mecanismo mucociliar alterado lo que predispone a mayor acumulo de secreciones y de infección. Son necesarias al menos 8 semanas de abstinencia para conseguir la disminución de riesgo de complicaciones al invertir algunos de los cambios epiteliales y de los valores de función pulmonar, como son los volúmenes de cierre, el calibre de las vías respiratorias pequeñas y el aclaramiento traqueobronquial de partículas. La EPOC en cirugía torácica tiene un riesgo 4.7 veces mayor con respecto a otra persona que no padezca tal patología, si se tiene en cuenta que se le va a realizar una lobectomía o neumonectomía a una persona con un pulmón que tiene otra patología pulmonar de base 6 .

e) La herida quirúrgica que se produce durante la resección pulmonar está considerada entre las más dolorosas, junto con las abdominales altas y las renales. El dolor posquirúrgico condicionará alteraciones en la mecáncica pulmonar, con disminución de la distensibilidad pulmonar y la instauración de un patrón respiratorio posquirúrgico poco profundo y con frecuencia respiratoria elevada, así como alteraciones en los mecanismos de defensa de la permeabilidad de la vía aérea, con fracaso del patrón tusígeno.

Se trata de dolor agudo iniciado por la lesión hística ocasionada por el bisturí sobre los tejidos. Pero además de este componente inflamatorio, existen otras fuentes de dolor como son ${ }^{12}$ :

- El uso de separado res para descubrir el pulmón, que puede producir lesiones en el paquete vásculonervioso intercostal que discurre por el borde inferior de la costilla superior. Estas lesiones producen disestesias del territorio afectado, con alteraciones de la sensibilidad del lado caudal que puede durar meses después de la intervención, así como hipotonía muscular de los músculos seccionados.

- La apertura importante del tórax puede dañar las articulaciones costovertebrales, costo transversales e interapofisiarias a nivel dorsal.

- Las cánulas de drenaje a nivel del espacio pleural son otra fuente de dolor ya que rozan el diafragma inhibiendo su movimiento, están en contacto con la pleura parietal (altamente inervada) y comprimen el nervio intercostal y perforan los músculos intercostales.

- En el caso de neumonectomías pueden aparecer dolores profundos, internos retroesternales con una probable relación a los desplazamientos del mediastino.

La intensidad disminuye con el proceso de cicatrización, si bien a largo plazo pueden permanecer dolores residuales posteriores a la resolución de la herida. El dolor posquirúrgico, además de suponer sufrimientos injustificados para el paciente, incrementa notablemente el riesgo de aparición de complicaciones posquirúrgicas, por lo que su control es uno de los retos más importantes en el posoperatorio. Se ha de insistir aquí en el hecho de que las toracotomías generan un patrón funcional posoperatorio restrictivo, con una disminución de hasta un 45-70 \% en la Capacidad Pulmonar Total. El temor que tiene el paciente de dañar la cirugía por el movimiento, hacen que disminuya la profundidad de 
la inspiración y que la tos se vuelva inefectiva, con el riesgo asociado de sufrir atelectasias y procesos infecciosos por retención de secreciones.

d) La ventilación mecánica invasiva es indispensable mientras el paciente requiera de un soporte ventilatorio que le permita mantener estable desde el punto de vista cardio-respiratorio y hemodinámico. La vía área artificial afecta los mecanismos de defensa del sistema respiratorio, alterando el mecanismo de aclaramiento mucociliar e inhibiendo la tos. Esto lleva a la retención de secreciones pudiendo producir obstrucciones totales de algún bronquio. Además una presión positiva continua de forma prolongada en un paciente con cirugía tóraco-pulmonar puede generar fugas de aire por los drenajes.

e) La anestesia general, así como los analgésicos, anestésicos y relajantes musculares, deprimen la función respiratoria, favoreciendo una respiración monótona y superficial, sin suspiros, con el consiguiente resultado en la formación de microatelectasias. Durante la anestesia general, las arelectasias aparecen rápidamente, como han demostrado estudios con Tomografía Axial Computerizada de tórax que muestran la existencia de atelectasias principalmente en las áreas dependientes del pulmón a los 5 minutos de la inducción. Además, la anestesia general puede exacerbar la producción de moco, por estímulo del tubo endotraqueal sobre las células mucosas y submucosas de la vía aérea y disminuir el mecanismo mucociliar. Estas alteraciones pueden extenderse hasta 6 días después de la cirugía, con presencia de secreciones anormalmente viscosas.

f) Fracción inspiratoria de óxigeno (FiO2): El suministro de oxígeno a niveles altos de $\mathrm{FiO} 2$ pueden generar atelectasias por desnitrogenización. Si permanece por un tiempo prolongado, superior a 6 horas, la hiperoxemia puede generar alteraciones celulares, principalmente en ellos neumocitos tipo II, perturbando así la secreción de surfactante y favoreciendo el colapso alveolar.

g) La posición operatoria: La limitación de la movilidad torácica en el plano de apoyo sobre la mesa quirúrgica y la mayor desinsuflación del pulmón infralateral favorecen, junto con la ventilación mecánica y la anestesia general, la aparición de atelecrasias.

La tabla 2 resume los principales cambios en la función pulmonar que se producen fruto de los diferentes factores de riesgo y que favorecen la aparición de complicaciones posquirúrgicas.

Tabla 2. Alteraciones en La función pulmonar

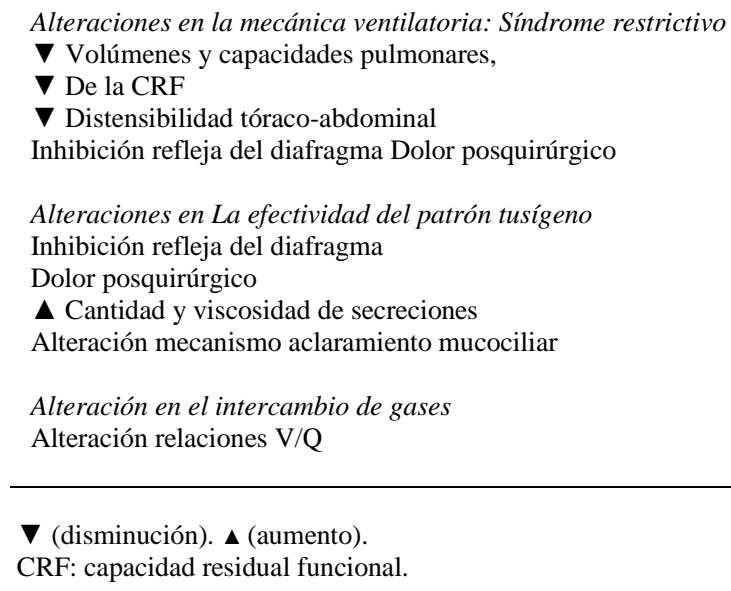

La tabla 3 recoge los criterios para la valoración del riesgo quirúrgico en varones adultos sometidos a neumonectomía ${ }^{13}$. Quienes cumplan estos criterios en la valoración funcional preoperatoria, tienen un riesgo de insuficiencia respiratoria posoperatoria o muerte mayor. Otro dato que se puede utilizar como predictivo de las complicaciones posoperatorias es el consumo de oxígeno máximo $\left(\mathrm{VO}_{2}\right)$ durante una prueba de ejercicio máximo. Para un $\mathrm{VO}_{2}<15 \mathrm{ml} / \mathrm{kg} / \mathrm{min}$, los índices de complicaciones aumentan. Si es inferior a $10 \mathrm{ml} / \mathrm{kg} / \mathrm{min}$, es más probable la muerte. La desaturación en ejercicio parece relacionarse también con alto riesgo de complicaciones cardiorrespiratorias ${ }^{14}$. 
Tabla 3. Valoración del riesgo quirúrgico en varones adultos sometidos a neumonectomía

1 VEMS $<2$ L. o del $50 \%$ de su valor de referencia (v.r)

2. MW $<50 \%$ v.r

3. DLco $<60 \%$

VEMS: volumen espiratorio máximo en el primer segundo; DLco: capacidad de difusión pulmonar del monóxido de carbono.

\section{Intervención de fisioterapia}

La intervención de fisioterapia debe de iniciarse en la fase preoperatoria de preparación, información y educación del paciente, y continuarse en la fase posoperatoria de restauración de la función pulmonar y prevención de las complicaciones respiratorias. En todo momento, se ha de buscar el hacer al paciente partícipe activo de su proceso de recuperación, facilitando su reincorporación al entorno social y familiar en el menor tiempo posible, disminuyendo de esta manera la estadía y los costes hospitalarios.

La tabla 4 recoge los objetivos de la Fisioterapia Respiratoria en las diferentes fases de la intervención.

Tabla 4. Objetivos de fisioterapia respiratoria

\begin{tabular}{lll}
\hline \multicolumn{1}{c}{ Fase prequirúrgica } & \multicolumn{1}{c}{ Fase posquirúrgica } \\
\cline { 2 - 3 } & & \multicolumn{1}{c}{ A corto plazo } \\
\hline $\begin{array}{l}\text { Aprendizaje de los procedimientos } \\
\text { terapéuticos }\end{array}$ & Alivio del dolor \\
$\begin{array}{l}\text { Mejora de la función respiratoria: } \\
\text { Mantenimiento de la permeabilidad de } \\
\text { Cón aérea }\end{array}$ & $\begin{array}{l}\text { Mantenimiento de una adecuada mecánica ventilatoria que } \\
\text { favorezca la reexpansión pulmonar y supla las demandas de } \mathrm{O}_{2} \\
\text { necesarias del metabolismo }\end{array}$ \\
& $\begin{array}{l}\text { Asegurar una buena la permeabilidad de la vía aérea } \\
\text { Efectuar el control postural y prevenir la aparición de } \\
\text { retracciones osteoarticulares en la cintura escapular }\end{array}$ \\
\hline
\end{tabular}

\section{Fase prequirúrgica}

Se ha demostrado que una correcta preparación e información de los pacientes, previa a la cirugía, disminuye la incidencia de complicaciones posoperarorias ${ }^{15}$, mejora su capacidad para realizar respiraciones profundas, reduce la necesidad de analgesia a la mitad de las dosis habituales y disminuye la estancia en el hospital ${ }^{16}$. La fase preoperatoria debe comenzar lo antes posible, tanto como la cirugía lo permita, con un objetivo doble: facilitar el aprendizaje de los procedimientos terapéuticos que el paciente deberá realizar después de la cirugía y mejorar la función respiratoria de manera previa al acto quirúrgico, con especial atención al mantenimiento de una vía aérea permeable. En el caso de las cirugías por cáncer de pulmón, los tiempos breves entre el diagnóstico del cáncer y la cirugía dificultan la participación de los pacientes en este tipo de programas de intervención prequirúrgica ${ }_{11}$.

Debe incluirse la evaluación clínica del enfermo basada en sus datos personales (edad, condición general, presencia de comorbilidades o factores de riesgo, pruebas de función pulmonar, características del tumor y tratamiento planeado), así como la evaluación funcional de la movilidad cervical y de la cintura escapular.

Además del aprendizaje del programa de fisioterapia que realizará en la fase posquirúrgica, se ha de aprovechar este momento para la práctica de la posición que mantendrá el paciente tras la cirugía, las trasferencias cama-silla y los movimientos de hombro y miembro superior del lado del pulmón operado antes de que se presente el dolor asociado a la toracotomía. 
En caso del que el paciente presente signos clínicos de un síndrome de encamamiento por inmovilización prolongada, se incorporarán actividades de cinesiterapia para prevenir el tromboembolismo pulmonar, la rigidez articular y la atrofia muscular.

En pacientes de alto riesgo, en los que se pueda anticipar la necesidad de utilizar una ventilación no invasiva en el posoperatorio, el paciente debería tomar contacto con este tipo de dispositivos antes de la cirugía y ajustar correctamente la máscara para familiarizados con los mismos ${ }^{2}$.

\section{Fase posquirúrgica}

Después de la cirugía torácica, la fisioterapia respiratoria es imprescindible. El desarrollo tecnológico y el tratamiento de pacientes progresivamente mayores hacen que cada vez se realice más cirugía en pacientes que están inmunocomprometidos o en pacientes que han recibido previamente radioterapia o quimioterapia para el trata- miento de su tumor. Es por ello, que se ha de poner más atención al período posoperatorio ya la prevención de las complicaciones que pueden aparecer en este momento.

\section{Alivio del dolor}

El grado de dolor que experimenta un paciente puede variar en intensidad, duración e incidencia en función de diferentes factores como son el tipo de intervención realizada, el nivel de umbral de dolor del paciente, la preparación prequirúrgica, la presencia de posibles complicaciones posquirúrgicas, el tratamiento anestésico y la calidad de los cuidados posoperatorios. Por ello, su valoración a lo largo del día a través de una escala analógica visual, graduada de 1 a 10, es una herramienta de gran utilidad para determinar los niveles de dolor y alcanzar los niveles óptimos de analgesia. El uso de anestesia regional y analgesia ha demostrado que disminuye o inhibe de manera significativa la respuesta de estrés neuroendocrina que afecta a la función cardiovascular, inmune y de coagulación después de la cirugía ${ }^{17}$.

El manejo del dolor posquirúrgico, comenzará por la reeducación postural en semisentado, evitando la posición antiálgica que adopta el paciente en cama, evitando los drenajes y cerrando el tórax intervenido. En relación a la posición, la que suele adoptarse es la de semisentado. Con relación a los decúbitos laterales podría utilizarse la posición de acostado sobre el lado no afecto o sano, por su contribución en la mejora de las relaciones ventilación/perfusión. No obstante, en el caso de las neumonectomias, hace años se consideraba una posición desaconsejable debido al riesgo que suponía de derrame de fluido en el interior de la sutura bronquial. Actualmente, el uso de suturas mecánicas elimina este riesgo, si bien la posición de decúbito lateral sobre el lado no afecto es mal tolerada por el paciente. El peso del líquido que ocupa el espacio dejado por el pulmón reseccionado, desplaza el medias tino sobre el pulmón y lo comprime, produciendo dificultad respiratoria al enfermo. El decúbito lateral sobre el lado de la neumonectomía es también mal tolerado por la presencia de la herida quirúrgica y los tubos de drenaje. La posición en trendelemburg está contraindicada.

Además de la reeducación postural, las medidas de contención de la incisión quirúrgica, la electroterapia analgésica, la cinesiterapia y la masoterapia, completan el abordaje terapéutico del dolor.

La contención manual de la incisión ha de realizarse en todas aquellas maniobras que determinen un incremento de la presión intratorácica e intraabdominal, como es el caso de la tos, principalmente, o de las variaciones de flujo aéreo. La asistencia debe realizarse a través de la aproximación manual de los bordes de la incisión, situando una mano posteriormente bajo la incisión de toracotomía, y otra mano anteriormente, evitando así la tracción sobre la sutura y disminuyendo el dolor, permitiendo maniobras de mayor eficacia. El propio paciente podrá efectuar autocontención de la herida ayudándose del apoyo del brazo homolateral a la incisión reforzado con la mano contralateral. El uso de esta técnica se mantendrá hasta que se alcance la cicatrización plena de la herida quirúrgica ${ }^{12}$ (fig. 6). 


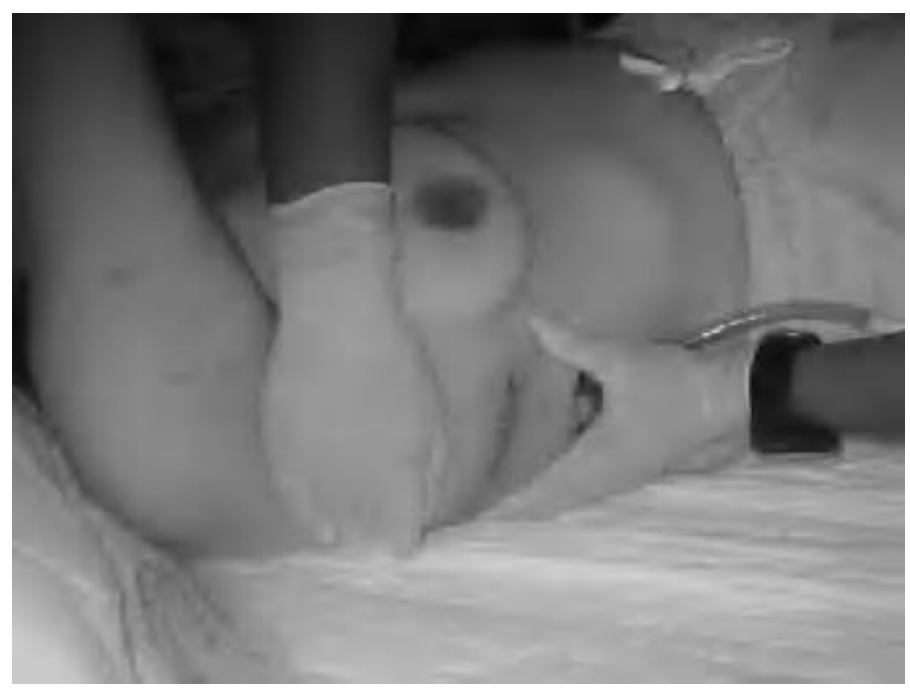

Fig. 6. Contención manual de la incisión realizada por el fisioterapeuta

La observación empírica de que determinadas formas de electroestimulación nerviosa transcutánea provocan analgesia es muy antigua, pero no será hasta el trabajo de Melzack y Wall de $1965^{18}$ que se proponga una explicación experimental sobre su posible mecanismo de acción. A partir de ese momento avanza notablemente el perfeccionamiento de las técnicas de aplicación, con la búsqueda de parámetros eléctricos, temporales y formas de aplicación de los electrodos que resulten más eficientes para obtener una respuesta analgésica en esta modalidad terapéutica.

La teoría antes citada, denominada "GATE CONTROL" (puerta de entrada), presenta un enfoque anatómico al basar su explicación en el comportamiento de las estructuras sensitivas del sistema nervioso central y periférico a la hora de conducir los estímulos. Aún, no está totalmente aclarado el mecanismo a través del cual la estimulación eléctrica transcutánea (TENS) alivia el dolor. Resumiendo la teoría de Melzack y Wall, se puede afirmar que la transmisión del dolor se produce desde la periferia a la médula espinal por medio de fibras tipo $\mathrm{C}$ de pequeño diámetro (amielínicas). Estas fibras excitan de manera directa o indirecta las células de transmisión en la médula espinal (interneuronas), que transmiten la información del dolor a los centros del dolor de los niveles más superiores en el cerebro a través del haz espinota- lámico lateral. Si un estímulo, sobreañadido al doloroso, excita las fibras de diámetro mayor $(\mathrm{A} \beta)$ que son más veloces en conducción e inervan los receptores cutáneos (tacto, presión), la transmisión sináptica entre las fibras del dolor y las células de transmisión queda inhibida, y de esta manera, no se percibe el dolor de forma consciente.

Diferentes autores ${ }^{19-22}$ han comprobado que el alivio del dolor obtenido por electroestimulación, en base a esta teoría, se mantiene mientras el estímulo eléctrico es capaz de despolarizar los receptores sensitivos inervados por fibras aferentes de grueso calibre. Cuando se produce acomodación de estos receptores o el estímulo eléctrico cesa, la percepción dolorosa se instaura de nuevo.

Esta forma de tratamiento se utiliza en el alivio del dolor de origen agudo a nivel de las afecciones músculo esqueléticas o en caso de dolor después de la cirugía.

La estimulación óptima de las fibras de grueso calibre (y por tanto el máximo alivio del dolor), se obtiene utilizando impulsos de duración corta y una intensidad que sea percibida por el paciente como una sensación de cosquilleo. La frecuencia de los estímulos conviene fijar- la en los niveles altos como $100 \mathrm{~Hz}$. Se evitará la aparición de contracciones musculares.

Según Marchand ${ }^{23}$, los pacientes con un consumo moderado de cafeína (aproximadamente $200 \mathrm{mg}$, dos o tres tazas de café) pueden ver disminuida la efectividad de la TENS. Ello es debido a que la cafeína compite con la adenosina, mediador primario del efecto analgésico de la TENS, por el sitio receptor. Al ocupar la cafeína sus receptores, la adenosina no puede alcanzar su correspondiente receptor y produce una disminución de la efectividad de la TENS.

Posteriormente, esta teoría del "GATE CONTROL" fue complementada por Castel ${ }^{24}$ exponiendo el papel que desempeñarían las "encefalinas" en el mecanismo de inhibición sináptica. Estas sustancias serían liberadas al producirse la estimulación de los receptores inervados por fibras mielínicas de diámetro grande a partir de pequeñas interneuronas encefalínicas situadas en el cuerno dorsal de la médula espinal, bloqueando la transmisión sináptica de la información del dolor al cerebro y evitando, consecuentemente, la percepción consciente del mismo. 
Sjolund y Ericsson en $1976^{25}$, explican los efectos analgésicos de la TEN S que se presentan de forma diferida, y con una duración variable como consecuencia de la electroestimulación, y que no podrían justificarse en la teoría del bloqueo de Melzack. La respuesta viene dada por la liberación de sustancias que ellos denominaron morfina endógena o endorfina. Estos opioides endógenos liberados como consecuencia de la estimulación actúan ocupando en los nociceptores los espacios reservados a los neurotransmisores algógenos impidiendo, así, la transmisión del impulso doloroso.

$\mathrm{Castel}^{24}$ propone una teoría, ya intuida por Melzack ${ }^{18}$, según la cual el control del dolor está localizado en el tallo encefálico, y ejerce una intensa influencia inhibitoria sobre la transmisión de impulsos que transportan información sobre el dolor. Estás áreas, están situadas principalmente en la materia gris periacueductal, así como en el núcleo del rafe, y son preferentemente activadas por fibras de diámetro reducido (fibras "C"). La estimulación eléctrica intensa de las fibras del dolor periféricas, por medio de TEN S de alta intensidad y baja frecuencia, o de técnicas de electroacupuntura tiene como resultado la "analgesia por hiperestimulación". Mientras que, en la TENS convencional la duración del alivio es, por regla general, reducida al tiempo de estimulación y un corto período posterior. Esto vendría justificado a causa de la vida media relativamente corta de las encefalinas. Sin embargo, la duración del alivio tras la "hiperestimulación" con TENS puede ser de días o semanas, y en algunos casos el alivio es permanente.

La metodología terapéutica basada en esta teoría, permite combatir el dolor profundo y crónico. También el dolor debido al daño por sobrefunción. Se emplean los estímulos eléctricos sobre las áreas dolorosas, puntos de acupuntura o puntos gatillo, situando uno de los electrodos en el punto doloroso y el otro a distancia del área dolorosa ${ }^{26}$.

Inicialmente se toma el electrodo activo, que en caso de emplear corriente de impulsos monofásicos debe ser el positivo, y después de elevar la intensidad hasta el umbral sensitivo; se explora por deslizamiento sobre la piel en busca de los puntos que resultan hipersensibles a la corriente. En estos puntos, se aplica el electrodo incrementando la intensidad al nivel máximo tolerado por el paciente.

El protocolo terapéutico incluye:

1. La intensidad de la corriente deberá ser alta aproximándose al nivel del dolor; la contracción muscular es aceptable.

2. La duración del impulso será de $200 \mu$ sg a 10 msg.

3. El número de impulsos por segundo será de 1 a 5.

4. Se emplearan impulsos con corriente de alto voltaje.

5. El estímulo durará de 30-45 sg.

6. La estimulación será aplicada sobre puntos gatillo o puntos de acupuntura.

7. El número de puntos a tratar estará en relación a la situación clínica.

8. Si el resultado del tratamiento es favorable el alivio del dolor puede ser de 6 o 7 horas.

9. El uso periódico de este tipo de estimulación da los mejoras resultados para aliviar el dolor y puede llegar a ceder.

Parece ser que el núcleo del rafe es una fuente descendente de control del dolor. Las neuronas descendentes que se originan en el núcleo del rafe y descienden por el tracto dorso lateral hasta niveles diversos de la médula espinal, son activadas por la estimulación eléctrica intensa. El tracto dorso lateral hace sinápsis en el asta posterior de la médula con las interneuronas encefalínicas. Las interneuronas, liberan encefalinas inhibiendo la transmisión sináptica de impulsos de las neuronas aferentes de segundo orden.

Paralelamente, a la vía seroadrenérgica antes descrita, existe una segunda vía descendente noradrenérgica $^{27}$. El papel de esta vía no es totalmente conocido. Además no se conoce si la vía noradrenérgica descendente inhibe directamente la sipnásis en el cuerno posterior de la médula o estimula la actividad de las neuronas encefalínicas.

Otro efecto de la hiperestimulación eléctrica es la emisión de otra sustancia polipeptídica ${ }^{28-32}$ similar al opio denominada "endorfina", que procede de la hipófisis anterior. Un extenso complejo molecular conocido como "ACTH-hipotropina $\beta$ ", se desdobla para producir ßendorfina (que es un péptido neuroactivador con potente efecto analgésico), ciertos tipos de encefalinas y ACTH. La liberación de ACTH (Hormona Adenosin Corticotropa) tiene como resultado la liberación de corticoesteroides de las glándulas suprarenales. Estas sustancias antiinflamatorias pueden ayudar a la reducción del dolor.

Las endorfinas pueden reducir el dolor localmente aumentando el ritmo de degradación de prostaglandinas y bradicinina. No obstante, su función más importante en la reducción del dolor crónico y agudo se realiza a través de la estimulación directa de las células del núcleo del rafe. Esta estimulación aumenta la actividad de los mecanismos de control del dolor descendentes que ya han sido comentados. Una hipótesis $^{27}$ sostiene que la ßendorfina se libera como respuesta a la estimulación intensa y de baja frecuencia (1 a $5 \mathrm{~Hz}$ ) de puntos de acupuntuta o impulsos activadores específicos. 
El procedimiento de electroestimulación basado en la teoría de la "hiperestimulación descendente" está dirigido al tratamiento del dolor crónico. Se emplean cortos tiempos de estimulación intensa para causar la activación del mecanismo neuronal descendente ${ }^{33}$.

El protocolo terapéutico incluye:

1. La intensidad de la corriente deberá ser muy alta, aproximándose al nivel del dolor; la contracción muscular no es deseable.

2. La duración del impulso será de $10 \mathrm{msg}$.

3. La frecuencia de impulsos será de $80 / \mathrm{sg}$.

4. El estímulo durará de $30 \mathrm{sg}-1 \mathrm{mn}$.

5 La estimulación será aplicada sobre puntos gatillo o puntos de acupuntura.

6. La selección y el número de puntos a tratar estará en relación a la superficie de tratamiento.

La aplicación de la TENS como medio de analgesia posoperatoria en pacientes sometidos a cirugía torácica ha sido bastante estudiada por diversos autores ${ }^{34}{ }_{-}^{37}$, pero la metodología de los trabajos es muy diversa por lo que resulta difícil concluir sobre el grado de efectividad de esta modalidad analgésica. Existen grandes diferencias entre los tiempos de aplicación, intensidad, frecuencia, duración del tiempo de impulso y tipo de cirugías. Aunque, en general, estos trabajos reflejan que la utilización de la TENS suele aportar algún beneficio, tanto en la reducción del dolor como en la recuperación funcional.

Rooney et al en $1983^{38}$; nos ofrecen un trabajo en el que el grupo de estudio y el grupo control están suficientemente homogeneizados, la técnica de aplicación de la TENS respeta con precisión los principios teóricos y el método de valoración de los resultados es suficientemente claro.

Estos autores suministran TENS a pacientes posquirúrgicos después de toracotomía por cáncer de pulmón, las cirugías fueron realizadas por el mismo cirujano. El grupo de casos estaba formado por 22 individuos, al igual que el grupo control. La edad de los 44 pacientes, hombres y mujeres, estaba entre 22 y 70 años. El protocolo de aplicación fue el siguiente:

- Frecuencia: 120 impulsos/mn.

- Tiempo de impulso: 0,2 msg.

- Intensidad: $14-20 \mathrm{~mA}$.

- Electrodos de $2 \times 2,5 \mathrm{~cm}$ autoadhesivos situados a nivel superior e inferior de la incisión.

Ambos grupos recibieron narcóticos a demanda en las primeras 24 horas del posoperatorio, estudiando la frecuencia de la misma durante este período. Los resultados obtenidos fueron los que se detallan en la tabla 5 .

Tabla 5. Narcóticos a demanda las $24 \mathrm{~h}$ del posoperatorio. Resultados obtenidos

\begin{tabular}{lll}
\hline & Casos $(n=22)$ & Controles $(n=22)$ \\
\hline Administración de Narcóticos & & \\
$24 \mathrm{~h}$ & $31,8 \%$ & $100 \%$ \\
$\quad$ Primeras o segundas 12 h & $45,5 \%$ & $100 \%$ \\
$\quad$ Ninguna & $22,7 \%$ & $100 \%$ \\
& & \\
Frecuencia de administración & & $68,2 \%$ \\
$\quad$ Cada 2-4 h & $41,2 \%$ & $31,8 \%$ \\
$\quad$ Cada 4-12 h & $58,8 \%$ & \\
& & \\
\hline
\end{tabular}

El 22,7 \% de los pacientes del grupo experimental no precisó narcóticos durante las primeras 24 h, lo cual resulta estadísticamente significativo. Las diferencias obtenidas en relación a la frecuencia de administración de narcóticos en ambos grupos no mostró significación estadística.

Según estos autores la TENS resulta un método no invasivo para el alivio del dolor en pacientes posquirúrgicos con cáncer de pulmón.

La masoterapia y la cinesiterapia a nivel de la cintura escapular, ráquis cervical y de la cicatriz permiten aliviar el dolor producido por las contracturas musculares secundarias a la sección del tejido 
muscular durante la cirugía y a la presencia de dolor. También alivia el dolor procedente de las articulaciones dorsales, sometidas a un estiramiento excesivo durante la cirugía. Estos elementos son los responsables a largo plazo de perpetuar el dolor posoperatorio, por lo que, la instauración precoz de estas técnicas de tratamiento contribuirá a su prevención (fig. 7).

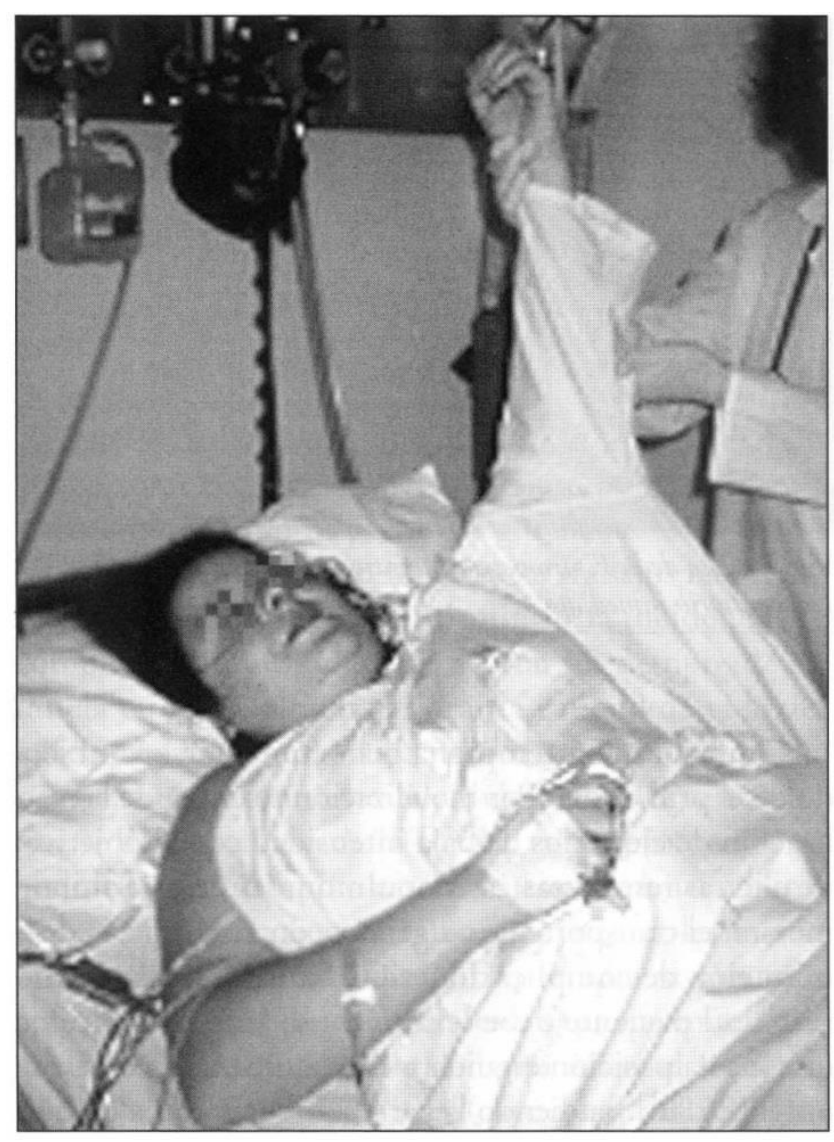

Fig.7. Cinesiterapia a nivel de la cintura escapular en el posoperatorio inmediato.

Las técnicas de masoterapia a realizar han de ser fundamentalmente de fricción a nivel paravertebral dorsal y de amasamiento en la cintura escapular, evitando acciones muy amplias alrededor de las cánula de drenaje. La cinesiterapia pasiva y activa implica a todas las articulaciones del raquis y cintura escapular, tratando de alcanzar los $90^{\circ}$ de abducción de hombro desde el primer momento y en caso de intubación del paciente, se aconseja movilizar el raquis suavemente en los rangos de movilidad que los dispositivos de intubación permitan. Los ejercicios activos, activo-asistidos y resistidos de cintura escapular a través de técnicas de facilitación neuromuscular deben comenzar una vez que el dolor lo permite desde el primer día de la intervención. Con ellos se mantiene el rango de movilidad y en ocasiones se observa una mejora en los valores de saturación de oxígeno ${ }^{2}$.

\section{Modificación de la mecánica ventilatoria: Mejora de la CRE incremento del volumen tidal y disminución} de la frecuencia respiratoria

Los ejercicios respiratorios deben comenzar el mismo día de la cirugía ${ }^{2}$; y en caso de haberse realizado, ha de reiniciarse el programa de tratamiento prequirúrgico.

En este momento inicial, los ejercicios respiratorios van encaminados a la prevención y control de las atelectasias, que son las complicaciones respiratorias más comunes presentes, en algún grado, en el $95 \%$ de los pacientes operados. Su causa hay que buscada en la falta de estímulo para la re-expansión pulmonar que impide restaurar la ventilación en los segmentos colapsados, más que en la presencia de tapones de moco. Esto es así debido al patrón restrictivo que se genera después de la cirugía, con una 
disminución de la CRF, y un paciente respirando con una frecuencia respiratoria elevada y un volumen corriente bajo. De ahí que los esfuerzos del fisioterapeuta en esta fase vayan dirigidos a mejorar el patrón ventilatorio del paciente y a mejorar la CRF a través de ejercicios de respiración profunda para expandir los alvéolos colapsados.

Los ejercicios de respiración profunda pueden realizarse con o sin el uso de Espirometría incentivada, si bien esta garantiza el trabajo del paciente a un volumen pulmonar situado entre el $35 \%$ y el $50 \%$ de la capacidad vital preoperatoria. Durante las 72 primeras horas después de la cirugía, las maniobras deben realizarse de forma intensiva (cada hora) ${ }^{13}$. A la hora de programar el volumen inspiratorio, hemos de considerar que una lobectomía conduce a los seis meses de la cirugía a una reducción adicional $\leq 10 \%$ de la Capacidad Vital Forzada; mientras que la resección de un pulmón completo generará una importante disminución de la reserva pulmonar del paciente, con una reducción permanente alrededor del 30-50 \% de la función pulmonar global ${ }^{13}$ (fig.8).

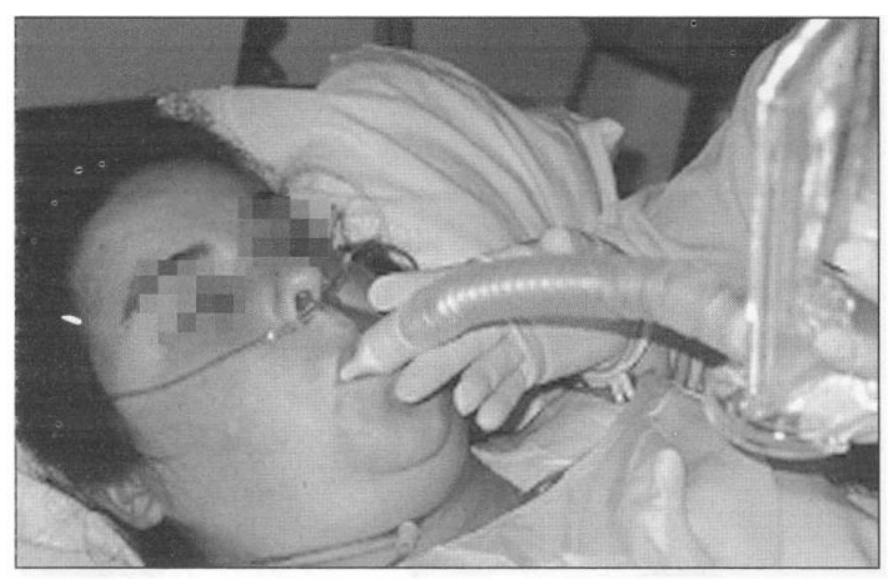

Fig. 8. Uso de la Espirometría lncentivada de Volumen en el posoperatorio inmediato.

El mantenimiento de una apnea teleinspiratoria de al menos 3 segundos hace las respiraciones profundas más efectivas en este proceso de reexpansión, ya que permiten que la ventilación se distribuya hacia los alvéolos con menor distensibilidad. Estos ejercicios pueden realizarse junto con otras técnicas de control respiratorio como son la respiración con labios fruncidos y la respiración diafragmática.

La movilización temprana debe fomentarse también desde el primer día. Por movilización se entiende la realización de ejercicios de baja intensidad con el objetivo de elicitar respuestas cardiopulmonares que permitan mejorar el transporte de oxígeno y contribuyan a la disminución de complicaciones pulmonares posoperatorias $^{39}$. El paciente debe levantarse lo antes posible, ya que la sola posición erguida, per se, aumenta la CRF en un 10-20 \% ${ }^{13}$, pudiendo llegar a subir escaleras a menudo a partir del tercer día de la operación $^{2}$. La marcha precoz mejora la CRF y la ventilación minuto, mejorando la ventilación sobre las unidades alveolares dependientes y en mayor riesgo de atelectasia. Sin embargo, para obtener estos efectos beneficiosos es necesario que la actividad física alcance una intensidad los suficientemente alta, cosa que con frecuencia no ocurre en la práctica clínica diaria, en la que el paciente camina a lo largo de un pasillo de acuerdo a límites muy subjetivos y en pocas ocasiones se usan medidas objetivas como son la frecuencia cardíaca, la frecuencia respiratoria, y la escala de Borg de percepción de la disnea, de uso común para la prescripción de la actividad física. Este es un punto que aún debe ser estudiado con más profundidad de cara a sacar el máximo beneficio terapéutico de la marcha en estos casos, de la manera más segura para el paciente. Por otro lado, considerando uno de los objetivos de la marcha el mejorar el volumen tidal, puede ser de utilidad indicar al paciente que durante la marcha realice respiraciones profundas 40

Independientemente de si la movilización alcanza o no los niveles de intensidad necesarios para modificar los patrones ventilatorios, contribuye a disminuir el desacondicionamiento muscular de los miembros inferiores, a prevenir las contracturas musculares y a mejorar la tolerancia ortostática ${ }^{41}$.

El plan de movilización temprana debe ser instaurado y monitorizado por el fisioterapeuta ${ }^{42}$, quién ha de coordinar estas acciones con el personal de enfermería de cara a obtener una mayor eficacia en la aplicación de los diferentes procedimientos terapéuticos. De esta manera, es posible aprovechar 
actividades que el paciente tiene que hacer durante el día, como puede ser el caminar al baño, el cambiar la posición del paciente de la cama a la silla para la hora de la comida, para realizar ciertos ejercicios de fisioterapia ${ }^{2} \bullet$

\section{Permeabilización de la vía aérea}

En el caso de que el paciente retenga secreciones o cuando hay signos de atelectasia o de colapso pulmonar, deben de utilizarse técnicas de eliminación de secreciones. Para ello utilizamos vibraciones y técnicas de variación de flujo aéreo, que mejoran las propiedades reológicas de las secreciones y facilitan su traslado. En el caso de las neumonectomias, las técnicas Ciclo Activo y Drenaje Autógeno son preferibles a la tos de cara a proteger la sutura bronquial, pues llevan asociados menores incrementos de las presiones intratorácicas ${ }^{7}$. En todo caso, no es conveniente efectuar espiraciones muy rápidas y forzadas, ante el riesgo de aparición de colapso alveolar.

Es interesante valorar el uso de vibraciones endógenas a través de dispositivos como el Flutter, Acapella y RC-Cornet, pues son menos dolorosas que las vibraciones manuales al no llevar asociado el componente de presión. No obstante, su uso debe realizarse con precaución al generar presiones positivas oscilatorias sobre la vía aérea, que pueden perpetuar o aumentar el escape de aire. Para minimizar este efecto, debemos utilizar siempre presiones bajas. La misma precaución ha de considerarse para el uso de Presiones Espiratorias Positivas, bien sea a través del sistema TheraPEP o de la máscara PEP. Estos dispositivos, además de ayudar a la eliminación de secreciones periféricas, incrementa la CRF contribuyendo en la lucha contra las atelectasias.

Si se produjo afectación del nervio laríngeo durante la cirugía, la efectividad de la tos puede alterarse con un aumento de la dificultad para eliminar secreciones. En estos casos, las técnicas de variación de flujo aéreo con glotis abierta son más recomendables que la tos, y en caso de retención de secreciones persistente, puede considerarse el utilizar técnicas de aspiración. El uso de técnicas de succión de secreciones ha de emplearse con el máximo cuidado para evitar el trauma sobre la sutura bronquial, especialmente si ha sido el pulmón derecho el resecado, debido a que la angulación de su bronquio principal facilita la entrada de la sonda de aspiración en el mismo ${ }^{7}$.

\section{Reentrenamiento al esfuerzo}

El entrenamiento global en el ejercicio, incluidos el de resistencia de las extremidades superiores e inferiores y de estiramientos, es un componente esencial en un programa global de rehabilitación pulmonar $^{13}$. La resección de un lóbulo pulmonar apenas conlleva repercusiones sobre la capacidad funcional para la actividad física del sujeto, sin embargo, la resección de un pulmón completo genera una reducción de un $20 \%$ en la capacidad de ejercicio, pudiendo presentar algunos pacientes disnea durante la actividad física ${ }^{7}$. Ello hace pensar que se trataría de candidatos susceptibles de entrar en un programa de rehabilitación pulmonar. No obstante, hay que señalar que los programas de rehabilitación pulmonar se han desarrollado y aplicado con éxito fundamentalmente en el entrenamiento de los pacientes con EPOC y en aquellos sometidos a cirugías de reducción de volumen, y si bien cabe suponer que en los pacientes sometidos a resección quirúrgica de pulmón por cáncer podrían obtenerse resultados beneficiosos, se carece de estudios suficientes que así lo confirmen. Desde un punto de vista funcional, hay que señalar que tras una neumonectomía el pulmón que queda es capaz de acomodar totalmente el flujo de sangre pulmonar, pero durante la rehabilitación pulmonar puede aparecer Hipertensión Pulmonar durante el ejercicio $^{7}$. A la hora de realizar el entrenamiento, en caso de que el paciente conserve los drenajes torácicos, es preferible realizar el ejercicio con cicloergómetro o un "step", para evitar los desplazamientos con los tubos de drenaje.

De manera complementaria, conviene enseñar al paciente aquellas adaptaciones que puede realizar en sus actividades de la vida diaria para conservar energía, especialmente si presenta disnea de esfuerzo.

\section{Conclusiones}

El posible papel de la rehabilitación pulmonar en la resección de cáncer de pulmón sigue siendo un área fértil que debe investigarse. Diferentes trabajos han mostrado su importancia para disminuir la incidencia de complicaciones respiratorias, acortar los tiempos de estancia en el hospital y mejorar la calidad de vida del enfermo. La fisioterapia dispone de modalidades terapéuticas que promueven el alivio del dolor, entre las que se destaca el uso de la TENS, cuyos protocolos en el dolor posquirúrgico están suficientemente definidos, si bien sería necesaria una mayor implantación clínica de su uso. Además, la fisioterapia respiratoria aporta un arsenal de procedimientos que permiten una adecuada permeabilización de la vía aérea, el aumento de los volúmenes pulmonares (capacidad funcional residual [CRF], capacidad 
vital $[\mathrm{CV}]$, capacidad inspiratoria $[\mathrm{CI}]$, volumen corriente $[\mathrm{VC})$ ) y permiten una actividad temprana optimizando la oxigenación y ventilación del paciente. Todo ello contribuye a la disminución del período de estancia media y los costes hospitalarios, permite a la persona una más rápida reintegración a su contexto social, con un bajo índice costo-beneficio. Recientemente un trabajo presentado en el Congreso Europeo de Cirugía Cardiotorácica por el Jefe de Servicio de Cirugía Torácica del Hospital Universitario de Salamanca, el profesor González Varela, demuestra que la inversión en fisioterapia a través de la contratación de profesionales de fisioterapia en estas unidades, ha conseguido reducir el número de complicaciones respiratorias posoperatorias tras lobectomía pulmonar, y disminuir la estancia media hospitalaria del paciente en dos días, así como el coste global de las cirugías, ahorrando entre 300 y 350 euros por cada lobecromía?.

\section{Agradecimientos}

Los autores de este artículo quieren mostrar su agradecimiento al Prof. Ursicino Martínez Rodríguez, así como al Dr. José M. ${ }^{a}$ Borro y a las Fisioterapeutas Esther Jiménez Moolhuyzen y Beatriz López Gabin, del servicio de cirugía torácica y rehabilitación pulmonar del Complejo Hospitalario Juan Canalejo-Marítimo de Oza de la ciudad de A Coruña, por su inestimable colaboración.

\section{Bibliografía}

1. Píriz Campos R, De la Fuente M. Enfermería Médico-Quirúrgica. Madrid: Editorial difusión avances de enfermería (DAE); 2001.

2. Ridley SC, Henil-Green A. Surgery for adults. En: Pryor JA, Ammani Prasad S, editors. Physiotherapy for Respiratory and Cardiac Problems. Adults and Paedriatics. London: Churchill Livinsgtone; 2002. p. 377-424.

3. Van Houte P, McDonald S, Yuang-Chi Chang A, Sal azar O. Cáncer de Pulmón. En: Rubin P, editor. Oncología Clínica. Enfoque mulridisciplinario para médicos y estudiantes. 3. ${ }^{a}$ ed. Madrid: Elsevier Science; 2003. p. 823-44.

4. Ovenrend TJ, Anderson CM, Lucy SD, Bhatia C, Jonsson BI, Timmermans C. The effect of Incentive Spiromerry on posto-perative pulmonary complications. Chest. 2001;120(3):971-8.

5. Mackay MR, Ellis E, Johnston C. Randomised clinical trial of physiotherapy after open abdominal surgery in high risk patients, Australian journal of Physiotherapy. 2005;51: 151-9.

6. Montemayor Rubio T, Ortega Ruiz F, Cejudo P. Rehabilitación respiratoria como profilaxis de complicaciones en cirugía mayor de pacientes con EPOC. Archivos de Bronconeumología. 2000; Supl 3):32-7.

7. Hough A. Physiotherapy in Respiratory Careo $3 .^{a}$ ed. London: Nelson Thomes Ltd, 2001.

8. Abeloff M, et al. Oncología clínica. Vol. n. 3. ${ }^{a}$ ed. Madrid: Editorial Elsevier; 2005.

9. Morén P. Intervenir en la contratación de fisioterapeutas ahorra costes y reduce complicaciones en las lobectomías. Diario Médico; 29/09/2005

10. Bozzone A, Romanelli A, Magrote G, Pascoli M, Milazzo M, Sterzi S. Pulmonary Rehabilitation: Pre- and postoperative treatmenr. RAYS. 2004;29(4):431-3.

11. Takaoka ST, Weinacker AB. The value of preoperative pulmonary rehabilitation. Thoracic surgery clinics. 2005; 15:203-11.

12. Valenza Derner G, González Doniz L, Yuste Sánchez MJ. Manual de Fisioterapia Respiratoria y cardíaca. Madrid: Editorial Síntesis; 2005. p. 157-81.

13. Celli BR. Rehabilitación en el paciente candidato a cirugía torácica. En: Güell Rous R, De Lucas Ramos P, editores. Tratado de rehabilitación respiratoria. Barcelona: Grupo Ars XXI de Comunicación; 2005. p. 279-88.

14. Puente-Maestu L, Ruiz Martin JJ. Las pruebas de esfuerzo en la cirugía de resección pulmonar. Arch Bronconeumol. 2003;39 (3): 126-32.

15. Clark CJ. Setting up a pulmonary Rehabilitation Programme. Thorax. 1994;49:270-8.

16. Egbert LD, et al. Reduction of postoperative pain by encouragement and instruction of patients. N Engl J Med. 1964;270:825-7.

17. Wu CL, Fleisher LA. Outcomes research in regional anesthesia and analgesia. Anestesia and Analgesia. 2000;91: 1232-42.

18. Melzack R, Wall P. Pain Mechanismes: a new theory. Sciencie. 1965; 150:917 -79.

19. Lampe G. Introduction to the use of transcutaneous electrical nerve stimulation devices. Phys Ther. 1978;58: 1504.

20. Bishop B. Pain: It's physiology and rationale for management. PhysTher.1980;60:13-37.

21. Melzack R, Stillwell D, Fox E. Trigger points and acupunrure points for pain: correlations and implications. Phys Ther. 1977;3(1):3-23.

22. Chesterton L, et al. Effects of TENS frecuency, intensity and stimulation site parameter manipulation on pressure pain thresholds in healthy human subjects. Pain. 2003;106:73-80.

23. Marchan S, et al. Letter to editor: Effects of cafeine on analgesia from transcutaneous electrical nerve stimularion. N Engl J Med. 1995;333:325.

24. Castel J. Pain management: acupunture and transcuraneus electrical nerve stimulation techniques; Lake Bluff, Illinois, 1979; Pain Control Services.

25. Sjolund B, Ericksson M. Electoacupunrure and endogenous morphines. Lancet. 1976. p. 1085.

26. Malizia E. Electroacupunture and peripheal $\beta$-endorphin and ACTH levels. Lancet. 1979;8:535-6. 
27. Jessell T, Kelly D. Pain and analgesia. En: Kandel E, Schwartz J, Jessell T, edirors. PrincipIes of neural sciencie. Norwalk, Connecticut, 1991.

28. Sjolund B, Ericksson M. Increased cerebroespinal fluid levels of endorphins after electro - acupunrure. Acta Physiol Scand. 1977;100:382-4.

29. Chapman C. Benedetti C. Analgesia flowing electrical stimulation: parcial reversal by narcotic anragonist. Life Sci. 1979;26:44-8.

30. Clement-Jons V, McLaughlin L, Toulin S. Increased beta - endorphin but not met - enkephalin levels in human cerebroespinal fluid after electroacupunture for recurrent pain. Lancer. 1980;2:946-8.

31. Pomeranz B, Paley D. Electro - acupuntura hipoalgesia is mediated by afferent impulses: an electrophysiological study in mice. Exp Neurol. 1979;66:398-402.

32. Salar G, Job I, Mingringo S. Effects of transcutaneous electro- therapy on CSF beta - endorphin content in patients without pain problems. Pain. 1981; 1 O: 169-72.

33. Castel J. Pain management with acupunture and transcutaneous electrical nerve stimulation techniques and photo stimulation (laser). Symposium on pain management, Walter Reed Army Medical Center; 1982.

34. Warfield C, Stein J, Howard A. The effect of transcutaneous Electrical Nerve stimulation on pain after thoracotomy, The Annals of Thoracic Surgery. 1985;39(5):463-5.

35. Benederri F, et al. Control postoperative Pain by Transcutaneous Electrical Nerve Stimulation after thoracic operations. The Annals of Thoracic Surgery. 1997;63:773-6.

36. Benederri F, et al. Postthoracoscopy Pain: Is TENS me answer. The Annals of Thoracic Surgery. 1997;64:890.

37. Yin-Ching Lin, et al. Effects of Transcutaneous Electrical Nerve Stimulation for post- thoracotomic pain. J Formosan Med Assoc. 1985;62:801-9.

38. Rooney Sh, et al. Effects of Transcutaneous Nerve Stimulation on postoperative pain after thoracotomy. Ansth Analg. 1983; 62:1010-4.

39. Zafiropoulos B, A1ison JA, McCarren B. Physiological responses to me early mobilisation of the intubared, ventila red abdominal surgery patient. Australian Journal of Physiotherapy. 2004;50:95-100.

40. Orfanos P, Ellis E, Johnston C. Effects of deep breathing exercises and ambulation on pattern of ventilation in post-operative patients. Australian Journal of Physiotherapy. 1999;45: 173-82.

41. Imle PC, Kemic N. Changes with immobility and methods of mobilization. En: Mackenzie CF, Imle PC, Ciesla N, editors. Chest Physiotherapy in the Intensive Care Unit. London: Williams and Wilkins; 1989. p. 188-214.

42. Mackay MR, Ellis E, johnsron C. Randomized clinical trial of physiotherapy after open abdominal surgery in high risk patienrs. Australian Journal of Physiotherapy. 2005;51: 151-9. 